\title{
SOME INTEGRAL INEQUALITIES FOR CONVEX FUNCTIONS
}

\author{
M. EMIN OZDEMIR, HAVVA KAVURMACI AND MERVE AVCI
}

\begin{abstract}
In this paper, by using some classical inequalities from the Theory of Inequality and integral identity, we establish two new general inequalities. Our results have some relationships with certain integral inequalities obtained by Sarikaya and Aktan.
\end{abstract}

\section{Introduction}

The following inequality is well known in the literature as the Hermite-Hadamard integral inequality:

$$
f\left(\frac{a+b}{2}\right) \leq \frac{1}{b-a} \int_{a}^{b} f(x) d x=\frac{f(a)+f(b)}{2}
$$

where $f: I \subset \mathbb{R} \rightarrow \mathbb{R}$ is a convex function on the interval $I$ of real numbers and $a, b \in I$ with $a<b$.

A function $f:[a, b] \subset \mathbb{R} \rightarrow \mathbb{R}$ is said to be convex if wherever $x, y \in[a ; b]$ and $t \in[0,1]$, the following inequality holds

$$
f(t x+(1-t) y) \leq t f(x)+(1-t) f(y)
$$

We say that $f$ is concave if $(-f)$ is convex. For recent results concerning Hermite-Hadamard's inequality for convex function, we refer to the readers [1], [2], [3].

In [1], Sarıkaya and Aktan obtained the following results concerning Hermite-Hadamard inequality for functions whose second derivative in absolute value is convex as follows:

Theorem 1. Let $I \subseteq \mathbb{R}$ be an open interval, $a, b \in I$ with $a<b$ and $f: I \rightarrow \mathbb{R}$ be a twice differentiable mapping such that $f^{\prime \prime}$ is integrable and $0 \leq \lambda \leq 1$ : If $\left|f^{\prime \prime}\right|$ is a convex function on $[a, b]$; then the following inequalities hold:

$$
\left|(\lambda-1) f\left(\frac{a+b}{2}\right)-\lambda \frac{f(a)+f(b)}{2}+\frac{1}{b-a} \int_{a}^{b} f(x) d x\right|
$$

Corresponding author: Havva Kavurmaci.

2010 Mathematics Subject Classification. Primary 26D10, $26 \mathrm{D} 15$.

Key words and phrases. Convex function, Hermite-Hadamard inequality, Power-Mean inequality. 


$$
\leq\left\{\begin{array}{rlrl}
\frac{b-a)^{2}}{12}\left[\left(\lambda^{4}+(1+\lambda)(1-\lambda)^{3}+\frac{5 \lambda-3}{4}\right)\left|f^{\prime \prime}(a)\right|\right. & \\
\left.+\left(\lambda^{4}+(2-\lambda) \lambda^{3}+\frac{1-3 \lambda}{4}\right)\left|f^{\prime \prime}(a)\right|\right], & & \text { for } 0 \leq \lambda \leq \frac{1}{2}, \\
\frac{(b-a)^{2}(3 \lambda-1)}{48}+\left[\left|f^{\prime \prime}(a)\right|+\left|f^{\prime \prime}(b)\right|\right], & \text { for } \frac{1}{2} \leq \lambda \leq 1 .
\end{array}\right.
$$

- (Midpoint inequality) In (1.1), if we choose $\lambda=0$ we have;

$$
\left|\frac{1}{b-a} \int_{a}^{b} f(x) d x-f\left(\frac{a+b}{2}\right)\right| \leq \frac{(b-a)^{2}}{24}\left[\frac{\left|f^{\prime \prime}(a)\right|+\left|f^{\prime \prime}(b)\right|}{2}\right] .
$$

- (Trapezoid inequality) In (1.1), if we choose $\lambda=1$ we have;

$$
\left.\mid \frac{f(a)+f(b)}{2}-\frac{1}{b-a} \int_{a}^{b} f(x) d x\right) \mid \leq \frac{(b-a)^{2}}{12}\left[\frac{\left|f^{\prime \prime}(a)\right|+\left|f^{\prime \prime}(b)\right|}{2}\right] .
$$

- In (1.1), if we choose $\lambda=\frac{1}{2}$ we have;

$$
\left|\frac{1}{b-a} \int_{a}^{b} f(x) d x-\frac{1}{2}\left[f\left(\frac{a+b}{2}\right)+\frac{f(a)+f(b)}{2}\right]\right| \leq \frac{(b-a)^{2}}{48}\left[\frac{\left|f^{\prime \prime}(a)\right|+\left|f^{\prime \prime}(b)\right|}{2}\right] .
$$

Theorem 2. Let $I \subseteq \mathbb{R}$ be an open interval, $a, b \in I$ with $a<b$ and $f: I \rightarrow \mathbb{R}$ be a twice differentiable mapping such that $f^{\prime \prime}$ is integrable and $0 \leq \lambda \leq 1$ : If $\left|f^{\prime \prime}\right|^{q}$ is a convex function on $[a, b]$, $q \geq 1$; then the following inequalities hold:

$$
\begin{aligned}
& \left|(\lambda-1) f\left(\frac{a+b}{2}\right)-\lambda \frac{f(a)+f(b)}{2}+\frac{1}{b-a} \int_{a}^{b} f(x) d x\right| \\
& \quad \leq\left\{\begin{array}{rr}
\frac{b-a)^{2}}{12}\left[\left(\lambda^{4}+(1+\lambda)(1-\lambda)^{3}+\frac{5 \lambda-3}{4}\right)\left|f^{\prime \prime}(a)\right|\right. & \\
\left.+\left(\lambda^{4}+(2-\lambda) \lambda^{3}+\frac{1-3 \lambda}{4}\right)\left|f^{\prime \prime}(a)\right|\right], & \text { for } 0 \leq \lambda \leq \frac{1}{2}, \\
\frac{(b-a)^{2}(3 \lambda-1)}{48}+\left[\left|f^{\prime \prime}(a)\right|+\left|f^{\prime \prime}(b)\right|\right], & \text { for } \frac{1}{2} \leq \lambda \leq 1 .
\end{array}\right.
\end{aligned}
$$

- (Midpoint inequality) In (1.1), if we choose $\lambda=0$ we have;

$$
\left|\frac{1}{b-a} \int_{a}^{b} f(x) d x-f\left(\frac{a+b}{2}\right)\right| \leq \frac{(b-a)^{2}}{24}\left[\frac{\left|f^{\prime \prime}(a)\right|+\left|f^{\prime \prime}(b)\right|}{2}\right] .
$$

- (Trapezoid inequality) In (1.1), if we choose $\lambda=1$ we have;

$$
\left.\mid \frac{f(a)+f(b)}{2}-\frac{1}{b-a} \int_{a}^{b} f(x) d x\right) \mid \leq \frac{(b-a)^{2}}{12}\left[\frac{\left|f^{\prime \prime}(a)\right|+\left|f^{\prime \prime}(b)\right|}{2}\right] .
$$

- In (1.1), if we choose $\lambda=\frac{1}{2}$ we have;

$$
\left|\frac{1}{b-a} \int_{a}^{b} f(x) d x-\frac{1}{2}\left[f\left(\frac{a+b}{2}\right)+\frac{f(a)+f(b)}{2}\right]\right| \leq \frac{(b-a)^{2}}{48}\left[\frac{\left|f^{\prime \prime}(a)\right|+\left|f^{\prime \prime}(b)\right|}{2}\right] .
$$


In this paper, we obtain some inequalities concerning Hermite-Hadamard integral inequality for functions whose second derivative in absolute value is convex.

\section{Hermite-Hadamard type inequalities for convex functions}

Now, we will give a new Lemma and then we establish two new generalizations of HermiteHadamard type inequality.

Lemma 1. Let $I \subset R$ be an open interval, $a, b \in I$ with $a<b$ and $f: I \rightarrow \mathbb{R}$ be twice differentiable mapping such that $f^{\prime \prime}$ is integrable and $0 \leq t, \lambda \leq 1$. Then the following identity holds:

$$
\begin{aligned}
& \frac{(b-a)^{2}}{2} \int_{0}^{1} m(t, \lambda) f^{\prime \prime}(a t+(1-t) b) d t \\
& \quad=(2 \lambda-1) f\left(\frac{a+b}{2}\right)-\lambda(f(a)+f(b))+\frac{1}{b-a} \int_{a}^{b} f(x) d x
\end{aligned}
$$

with

$$
m(t, \lambda)= \begin{cases}t(t-2 \lambda), & t \in\left[0, \frac{1}{2}\right), \\ (1-t)(1-t-2 \lambda), & t \in\left[\frac{1}{2}, 1\right] .\end{cases}
$$

Proof. It suffices to note that

$$
\begin{aligned}
I= & \int_{0}^{1} m(t, \lambda) f^{\prime \prime}(a t+(1-t) b) d t \\
= & \int_{0}^{\frac{1}{2}} t(t-2 \lambda) f^{\prime \prime}(a t+(1-t) b) d t \\
& +\int_{\frac{1}{2}}^{1}(1-t)(1-t-2 \lambda) f^{\prime \prime}(a t+(1-t) b) d t \\
= & \left.\left(t^{2}-2 \lambda t\right) \frac{f^{\prime}(a t+(1-t) b)}{a-b}\right|_{0} ^{\frac{1}{2}}-\int_{0}^{\frac{1}{2}}(2 t-2 \lambda) \frac{f^{\prime}(a t+(1-t) b)}{a-b} d t \\
= & -\frac{2}{a-b}\left[\int_{0}^{\frac{1}{2}}(t-\lambda) f^{\prime}(a t+(1-t) b) d t+\int_{\frac{1}{2}}^{1}(t+\lambda-1) f^{\prime}(a t+(1-t) b) d t\right] \\
= & -\frac{2}{a-b}\left[\left.(t-\lambda) \frac{f(a t+(1-t) b)}{a-b}\right|_{0} ^{\frac{1}{2}}-\int_{0}^{\frac{1}{2}} \frac{f(a t+(1-t) b)}{a-b} d t\right. \\
& \left.\left.(t+\lambda-1) \frac{f(a t+(1-t) b)}{a-b}\right|_{\frac{1}{2}} ^{1}-\int_{\frac{1}{2}}^{1} \frac{f(a t+(1-t) b)}{a-b} d t\right] \\
= & -\frac{2}{(a-b)^{2}}\left[(1-2 \lambda) f\left(\frac{a+b}{2}\right)+\lambda(f(a)+f(b))\right]+\frac{2}{(b-a)^{3}} \int_{a}^{b} f(x) d x .
\end{aligned}
$$

By multiplying the $I$ with $\frac{(b-a)^{2}}{2}$, we get the desired equality.

Now by using the above lemma, we prove our general theorems: 
Theorem 3. Let $I \subset R$ be an open interval, $a, b \in I$ with $a<b$ and $f: I \rightarrow \mathbb{R}$ be twice differentiable mapping such that $f^{\prime \prime}$ is integrable and $0 \leq t, \lambda \leq 1$. If $\left|f^{\prime \prime}\right|$ is a convex function on $[a, b]$; then the following inequalities hold:

$$
\begin{aligned}
& \left|(2 \lambda-1) f\left(\frac{a+b}{2}\right)-\lambda(f(a)+f(b))+\frac{1}{b-a} \int_{a}^{b} f(x) d x\right| \\
& \quad= \begin{cases}\frac{(b-a)^{2}\left(64 \lambda^{3}-6 \lambda+1\right)}{48}\left[\left|f^{\prime \prime}(a)\right|+\left|f^{\prime \prime}(b)\right|\right], & \lambda \in\left[0, \frac{1}{4}\right), \\
\frac{(b-a)^{2}(6 \lambda-1)}{48}\left[\left|f^{\prime \prime}(a)\right|+\left|f^{\prime \prime}(b)\right|\right], & t \in\left[\frac{1}{4}, \frac{1}{2}\right] .\end{cases}
\end{aligned}
$$

Proof. From Lemma 1 and using the modulus, we get

$$
\begin{gathered}
\left|(2 \lambda-1) f\left(\frac{a+b}{2}\right)-\lambda(f(a)+f(b))+\frac{1}{b-a} \int_{a}^{b} f(x) d x\right| \\
\leq \frac{(b-a)^{2}}{2} \int_{0}^{\frac{1}{2}}|t(t-2 \lambda)|\left|f^{\prime \prime}(a t+(1-t) b)\right| d t \\
\quad+\int_{\frac{1}{2}}^{1}|(1-t)(1-t-2 \lambda)|\left|f^{\prime \prime}(a t+(1-t) b)\right| d t .
\end{gathered}
$$

We suppose that $2 \lambda \in\left[0, \frac{1}{2}\right)$ and using the convexity of $\left|f^{\prime \prime}\right|$, we get

$$
\begin{aligned}
& \frac{(b-a)^{2}}{2} \int_{0}^{\frac{1}{2}}|t(t-2 \lambda)|\left|f^{\prime \prime}(a t+(1-t) b)\right| d t \\
& \quad+\int_{\frac{1}{2}}^{1}|(1-t)(1-t-2 \lambda)|\left|f^{\prime \prime}(a t+(1-t) b)\right| d t \\
& \leq \frac{(b-a)^{2}}{2}\left\{\int_{0}^{2 \lambda}\left(2 \lambda t-t^{2}\right)\left[t\left|f^{\prime \prime}(a)\right|+(1-t)\left|f^{\prime \prime}(b)\right|\right] d t\right. \\
& \quad+\int_{2 \lambda}^{\frac{1}{2}}\left(t^{2}-2 \lambda t\right)\left[t\left|f^{\prime \prime}(a)\right|+(1-t)\left|f^{\prime \prime}(b)\right|\right] d t \\
& \quad+\int_{\frac{1}{2}}^{1-2 \lambda}\left(t^{2}+(2 \lambda-2) t+(1-2 \lambda)\right)\left[t\left|f^{\prime \prime}(a)\right|+(1-t)\left|f^{\prime \prime}(b)\right|\right] d t \\
& \left.\quad+\int_{1-2 \lambda}^{1}\left(-t^{2}+(2-2 \lambda) t+2 \lambda-1\right)\left[t\left|f^{\prime \prime}(a)\right|+(1-t)\left|f^{\prime \prime}(b)\right|\right] d t\right\} \\
& \leq \\
& \quad \frac{(b-a)^{2}\left(64 \lambda^{3}-6 \lambda+1\right)}{48}\left[\left|f^{\prime \prime}(a)\right|+\left|f^{\prime \prime}(b)\right|\right] .
\end{aligned}
$$

On the other hand, $2 \lambda \in\left[\frac{1}{2}, 1\right]$. And similarly using the convexity of $\left|f^{\prime \prime}\right|$, we get

$$
\begin{aligned}
& \frac{(b-a)^{2}}{2} \int_{0}^{\frac{1}{2}}|t(t-2 \lambda)|\left|f^{\prime \prime}(a t+(1-t) b)\right| d t \\
& \quad+\int_{\frac{1}{2}}^{1}|(1-t)(1-t-2 \lambda)|\left|f^{\prime \prime}(a t+(1-t) b)\right| d t \\
& \leq \frac{(b-a)^{2}}{2}\left\{\int_{0}^{\frac{1}{2}}\left(2 \lambda t-t^{2}\right)\left[t\left|f^{\prime \prime}(a)\right|+(1-t)\left|f^{\prime \prime}(b)\right|\right] d t\right.
\end{aligned}
$$




$$
\begin{aligned}
& +\int_{\frac{1}{2}}^{1}\left(-t^{2}+(2-2 \lambda) t+(2 \lambda-1)\right)\left[t\left|f^{\prime \prime}(a)\right|+(1-t)\left|f^{\prime \prime}(b)\right|\right] d t \\
\leq & \frac{(b-a)^{2}(6 \lambda-1)}{48}\left[\left|f^{\prime \prime}(a)\right|+\left|f^{\prime \prime}(b)\right|\right] .
\end{aligned}
$$

Then we use (2.2) and (2.3) in (2.1), we get the desired representation.

Remark 1. In Theorem 3, if we use $\lambda=0, \frac{1}{2}, \frac{1}{6}$; we have the inequalities (1.2), (1.3) and (1.4) respectively.

Theorem 4. Let $I \subset \mathbb{R}$ be an open interval, $a, b \in I$ with $a<b$ and $f: I \rightarrow \mathbb{R}$ be twice differentiable mapping such that $f^{\prime \prime}$ is integrable and $0 \leq t, \lambda \leq 1$. If $\left|f^{\prime \prime}\right|^{q}$ is a convex function on $[a, b]$, $q \geq 1$, then the following inequalities hold:

$$
\begin{aligned}
& \left|(2 \lambda-1) f\left(\frac{a+b}{2}\right)-\lambda(f(a)+f(b))+\frac{1}{b-a} \int_{a}^{b} f(x) d x\right| \\
& \quad= \begin{cases}\frac{(b-a)^{2}}{2}\left\{( \frac { 8 \lambda ^ { 3 } } { 3 } + \frac { 1 - 6 \lambda } { 2 4 } ) ^ { 1 - 1 / q } \left[\left(\frac{3-16 \lambda}{192}+\frac{8 \lambda^{4}}{3}\right)\left|f^{\prime \prime}(a)\right|^{q}\right.\right. \\
\left.+\left(\frac{8 \lambda^{3}(1-\lambda)}{3}+\frac{5-32 \lambda}{192}\right)\left|f^{\prime \prime}(b)\right|^{q}\right]^{\frac{1}{q}} \\
\left.+\left(\frac{(1-2 \lambda)^{2}(2 \lambda+2)}{3}+\frac{14 \lambda^{4}-5}{8}\right)^{1-1}\right)^{q}\left[\left((1-2 \lambda)^{3}\left(\frac{2 \lambda+1}{6}\right)+\frac{4 \lambda-1}{8}-\frac{1}{64}\right)\left|f^{\prime \prime}(a)\right|^{q}\right. & \\
\left.\left.\left((1-2 \lambda)^{2}\left(\frac{4 \lambda^{2}+4 \lambda+3}{6}\right)+\frac{5 \lambda-2}{4}+\frac{1}{64}\right)\left|f^{\prime \prime}(b)\right|^{q}\right]^{\frac{1}{q}}\right\}, & \lambda \in\left[0, \frac{1}{4}\right), \\
\frac{(b-a)^{2}}{2}\left(\frac{6 \lambda-1}{24}\right)^{1-\frac{1}{q}}\left\{\left[\frac{16 \lambda-3}{192}\left|f^{\prime \prime}(a)\right|^{q}+\frac{32 \lambda-5}{192}\left|f^{\prime \prime}(b)\right|^{q}\right]^{1 / q},\right. & t \in\left[\frac{1}{4}, 1\right] . \\
\left.+\left[\left(\frac{4 \lambda-1}{24}+\frac{1}{64}\right)\left|f^{\prime \prime}(a)\right|^{q}+\left(\frac{\lambda}{12}-\frac{1}{64}\right)\left|f^{\prime \prime}(b)\right|^{q}\right]^{\frac{1}{q}}\right\}, & \end{cases}
\end{aligned}
$$

Proof. Suppose that $q \geq 1$. From Lemma 1 and using the well-known power-mean inequality, we have

$$
\begin{aligned}
\left|(2 \lambda-1) f\left(\frac{a+b}{2}\right)-\lambda(f(a)+f(b))+\frac{1}{b-a} \int_{a}^{b} f(x) d x\right| \\
\leq \frac{(b-a)^{2}}{2}\left\{\left(\int_{0}^{\frac{1}{2}}|t(t-2 \lambda)| d t\right)^{1-\frac{1}{q}}\left(\int_{0}^{\frac{1}{2}}|t(t-2 \lambda)|\left|f^{\prime \prime}(a t+(1-t) b)\right|^{q} d t\right)^{\frac{1}{q}}\right. \\
\left.\quad+\left(\int_{\frac{1}{2}}^{1}|(1-t)(1-t-2 \lambda)| d t\right)^{1-\frac{1}{q}}\left(\int_{\frac{1}{2}}^{1}|(1-t)(1-t-2 \lambda)|\left|f^{\prime \prime}(a t+(1-t) b)\right|^{q} d t\right)^{\frac{1}{q}}\right\} .
\end{aligned}
$$

Let $2 \lambda \in\left[0, \frac{1}{2}\right]$. By a simple calculation, we have

$$
\begin{aligned}
\int_{0}^{\frac{1}{2}}|t(t-2 \lambda)| d t & =\frac{8 \lambda^{3}}{3}+\frac{1-6 \lambda}{24} \\
\int_{\frac{1}{2}}^{1}|(1-t)(1-t-2 \lambda)| d t & =\frac{(1-2 \lambda)^{2}(2 \lambda+2)}{3}+\frac{14 \lambda-5}{8} .
\end{aligned}
$$

And by using the convexity of $\left|f^{\prime \prime}\right|^{q}$, we have

$$
\int_{0}^{\frac{1}{2}}|t(t-2 \lambda)|\left|f^{\prime \prime}(a t+(1-t) b)\right|^{q} d t
$$




$$
=\left(\frac{3 \lambda-16}{192}+\frac{8 \lambda^{4}}{3}\right)\left|f^{\prime \prime}(a)\right|^{q}+\left(\frac{8 \lambda^{3}(1-\lambda)}{3}+\frac{532 l}{192}\right)\left|f^{\prime \prime}(b)\right|^{q}
$$

and

$$
\begin{aligned}
\int_{\frac{1}{2}}^{1} \mid & \left.(1-t)(1-t-2 \lambda)|| f^{\prime \prime}(a t+(1-t) b)\right|^{q} d t \\
\leq & \left((1-2 \lambda)^{3}\left(\frac{2 \lambda+1}{6}\right)+\frac{4 \lambda-1}{8}-\frac{1}{64}\right)\left|f^{\prime \prime}(a)\right|^{q} \\
& +\left((1-2 \lambda)^{2}\left(\frac{4 \lambda^{2}+4 \lambda+3}{6}\right)+\frac{5 \lambda-2}{4}+\frac{1}{64}\right)\left|f^{\prime \prime}(b)\right|^{q} .
\end{aligned}
$$

If we use (2.6)-(2.9) in (2.5), we get the first inequality in (2.4).

Now, let $2 \lambda \in\left[\frac{1}{2}, 1\right]$ : Similarly using the convexity of $\left|f^{\prime \prime}\right|$ and well-known power-mean inequality, we have

$$
\begin{aligned}
\mid(2 \lambda- & 1) f\left(\frac{a+b}{2}\right)-\lambda(f(a)+f(b))+\frac{1}{b-a} \int_{a}^{b} f(x) d x \mid \\
\leq & \frac{(b-a)^{2}}{2}\left\{\left(\int_{0}^{\frac{1}{2}}|t(t-2 \lambda)| d t\right)^{1-\frac{1}{q}}\left(\int_{0}^{\frac{1}{2}}|t(t-2 \lambda)|\left|f^{\prime \prime}(a t+(1-t) b)\right|^{q} d t\right)^{\frac{1}{q}}\right. \\
& \left.+\left(\int_{\frac{1}{2}}^{1}|(1-t)(1-t-2 \lambda)| d t\right)^{1-\frac{1}{q}}\left(\int_{\frac{1}{2}}^{1}|(1-t)(1-t-2 \lambda)|\left|f^{\prime \prime}(a t+(1-t) b)\right|^{q} d t\right)^{\frac{1}{q}}\right\} .
\end{aligned}
$$

A simple calculation, we have

$$
\int_{0}^{\frac{1}{2}}|t(t-2) \lambda| d t=\int_{\frac{1}{2}}^{1}|(1-t)(1-t-2 \lambda)| d t=\frac{6 \lambda-1}{24}
$$

and

$$
\begin{gathered}
\int_{0}^{\frac{1}{2}}|t(t-2) \lambda|\left|f^{\prime \prime}(a t+(1-t) b)\right|^{q} d t \leq \frac{6 \lambda-3}{192}\left|f^{\prime \prime}(a)\right|^{q}+\frac{32 \lambda-5}{192}\left|f^{\prime \prime}(b)\right|^{q} \\
\int_{\frac{1}{2}}^{1}|(1-t)(1-t-2 \lambda)|\left|f^{\prime \prime}(a t+(1-t) b)\right|^{q} d t \leq\left[\frac{4 \lambda-1}{24}+\frac{1}{64}\right]\left|f^{\prime \prime}(a)\right|^{q}+\left[\frac{\lambda}{12}-\frac{1}{64}\right]\left|f^{\prime \prime}(b)\right|^{q}
\end{gathered}
$$

If we use (2.11)-(2.13) in (2.10), we have the second inequality in (2.4). This completes the proof.

Remark 2. In Theorem 4 , if we use $\lambda=0, \frac{1}{2}, \frac{1}{6}$; we have the inequalities (1.5), (1.6) and (1.7) respectively.

\section{References}

[1] M. Zeki Sarıkaya, N. Aktan, On the generalization some integral inequalities and their applications, Mathematical and Computer Modelling, 54 (2011), 2175-2182.

[2] A. Sağlam, M. Zeki Sarıkaya and H. Yıdırım, Some new inequalities of Hermite-Hadamard.s type, Kyungpook Mathematical Journal, 50(2010), 399-410. 
[3] M. Zeki Sarıkaya, A. Sağlam and H. Yıdırım, Some new integral inequalities for twice differentiable convex mappings, arXiv:1005.0453v1 [math. CA].

Ataturk University, K.K. Education Faculty, Department Of Mathematics, 25240, Campus, Erzurum, Turkey.

E-mail: emos@atauni.edu.tr

Ataturk University, K.K. Education Faculty, Department Of Mathematics, 25240, Campus, Erzurum, Turkey.

E-mail: hkavurmaci@atauni.edu.tr

Adiyaman University, Faculty Of Science and Arts, Department of Mathematics, 02040, Adiyaman, Turkey.

E-mail: mavci@posta.adiyaman.edu.tr 